\title{
Kernos
}

Revue internationale et pluridisciplinaire de religion grecque antique

$21 \mid 2008$

Varia

\section{Natale SPINETO, Dionysos a teatro. Il contesto festivo del dramma greco}

Isabelle Tassignon

\section{OpenEdition \\ Journals}

\section{Édition électronique}

URL : https://journals.openedition.org/kernos/1690

DOI : 10.4000/kernos. 1690

ISSN : 2034-7871

\section{Éditeur}

Centre international d'étude de la religion grecque antique

\section{Édition imprimée}

Date de publication : 1 janvier 2008

Pagination : 333-335

ISSN : 0776-3824

Référence électronique

Isabelle Tassignon, « Natale spineto, Dionysos a teatro. II contesto festivo del dramma greco », Kernos [En ligne], 21 | 2008, mis en ligne le 15 septembre 2011, consulté le 24 août 2022. URL : http:// journals.openedition.org/kernos/1690; DOI : https://doi.org/10.4000/kernos.1690 
des résultats obtenus. L'érudition et la sagacité de l'A., déployées déjà dans une étude sur l'Apollon de Claros, n'avaient pas échappé du reste aux lecteurs de Kernos (16, 2003).

On soulignera aussi l'intérêt des réflexions méthodologiques que comporte l'ouvrage, tout spécialement dans la longue introduction : l'ouverture de l'A. à la méthode comparative, sa position nuancée concernant la possibilité d'invariants anthropologiques, mais, plus encore, les idées qu'il défend quant à la manière d'explorer le polythéisme ancien ainsi que le débat déjà ancien qu’il ranime à ce propos. G. Dumézil, Cl. Lévi-Strauss, M. Delcourt, J.-P. Vernant et M. Detienne sont les principaux maitres dont il se réclame. On le voit, comme ce dernier, travailler sur une micro-configuration, telle que l'esquissent en l'occurrence deux vers seulement d'un hymne, et se concentrer sur les modes d'action d'un dieu et sur les objets qu'ils mettent en œuvre, se servant d'eux comme d'un réactif pour faire apparaitre certaines facettes du dieu. Mais il est au moins deux points par lesquels il se distancie ici de son modèle. D’une part, dépassant l'espèce de nominalisme théologique qui se refuse à admettre derrière le nom d'un dieu une quelconque essence, Ph.M est convaincu que son enquête à mis au jour non seulement quelques traits sous lesquels Apollon est apparu parfois, mais bien un «noyau organisateur», un «substrat intangible» ou, pour mieux dire encore, son "être profond» (l'expression est de Marie Delcourt). D'autre part, - les deux positions se tiennent, - il estime qu'un dieu grec peut être envisagé en dehors de sa relation avec les autres dieux, même si l'examen de celle-ci n'est certes pas à dédaigner.

Ce n'est pas ici le lieu d'arbitrer pareil débat. "La voie d'un certain bon sens ne seraitelle pas médiane ? ", demandait naguère V. Pirenne-Delforge (Les panthéons des cités, Kernos, suppl. 8, 1998, p. 8) ? Souhaitons en tout cas que le dialogue se poursuive et qu'entrent aussi en scène les auteurs d'autres monographies récentes consacrées à un dieu grec, comme il en est plusieurs dans les suppléments à Kernos.

André MOTTE

(Université de Liège)

Natale SPINETO, Dionysos a teatro. Il contesto festivo del dramma greco, Roma, "L'Erma » di Bretschneider, 2005. 1 vol. $17 \times 24 \mathrm{~cm}$, XII+436 p. (Storia delle religioni, 16). ISBN : 88-8265-321-8.

Quels sont les mécanismes qui régissent la fête dionysiaque, en quoi chacune des quatre célébrations majeures que sont les Anthestéries, les Lénéennes, les Grandes Dionysies et les Dionysies aux champs se distinguent-elles l'une de l'autre, quelles sont leurs dynamiques individuelles et pour atteindre quel but? Quels groupes sociaux y participent, selon quelles modalités et lesquels en sont écartés ? Tel est le propos de l'ouvrage, dense et savant, de Natale Spineto. Alors que le classique Dramatic Festivals of Athens d'A. Pickard-Cambridge, publié à Oxford en 1953, envisageait les textes et la documentation iconographique pour tenter de reconstituer point par point, chronologiquement, le minutieux déroulement de chacune de ces fêtes, l'approche de N. Spineto n'a pas de dimension iconographique et privilégie un éclairage sociologique des sources littéraires.

Dans le cadre spatio-temporel de l'Athènes de la fin de l'époque archaïque et de l'époque classique, - une société globalement stable à de multiples points de vue -, l'A. étudie, pour chacune des fêtes susmentionnées, les participants (comment ils se définissent dans leur époque et les uns par rapport aux autres), le rituel, les mythes qui les sous-tendent et leur finalité. Selon l'A., toutes ces fêtes dionysiaques réactualisent en les contrôlant des situations d'anomie ou de suspension de la normalité. Première fête dionysiaque de l'année, les Anthestéries sont étroitement associées au calendrier agricole; elles célèbreraient l'ouverture des jarres à vin (la tradition, incarnée par A. Pickard-Cambridge et d'autres, rattachait 
les représentations de femmes ouvrant des jarres aux Lénéennes) et la possibilité de le boire. Le vin, les rapports entre vivants et morts, l'univers chaotique d'après le déluge (avec l'offrande de panspermia) sont les trois registres fondamentaux autour desquels se réactualiseraient rituellement des situations de désordre "pré-cosmique ». Les Lénéennes sont d'interprétation plus délicate encore. Selon l'A., le nom même de Lenaia implique une forme de violence portée sur le vin et sur Dionysos (et évoquent plus particulièrement un épisode de démembrement du dieu); elles mettent cependant en œuvre la même dynamique que les Anthestéries puisque l'on y trouve à nouveau comme principaux composants de ces fêtes une situation d'anomie, matérialisée par une procession, et des allusions aux relations entre vivants et morts. L'embarras de l'A. est ici perceptible notamment lorsqu'il s'agit de déterminer à laquelle des deux fêtes appartiennent l'ouverture des jarres et les rites accomplis par les femmes devant le masque divin. Dans le cas des Lénéennes comme dans celui des Anthestéries, la période historique de leur fondation ne peut pas être déterminée. Enfin, les Dionysies aux champs, célébrées en décembre-janvier, sont les dernières de ce trio de fêtes " paysannes ». Pour l'A., il s'agit probablement de fêtes de création plus récente que les trois autres, mais elles suivent le même schéma avec une phallophorie, - évoquant à nouveau un état de suspension de la normalité -, à laquelle participent des groupes sociaux marginaux (femmes, étrangers, esclaves).

Les Grandes Dionysies, auxquelles l'A. a consacré un gros chapitre, sont des fêtes à la dimension sociale et politique très marquée. Ces fêtes sont aussi plus récentes que les Anthestéries et que les Lénéennes, et sont généralement associées à l'époque de Pisistrate. La question de savoir si ces fêtes ont été créées par Pisistrate ou si le tyran d'Athènes aurait régénéré une fête plus ancienne est délicate. Sans rejeter tout à fait la première, l'A. privilégie la seconde hypothèse - les ressemblances structurelles avec les Dionysies aux champs semblent plaider en faveur d'une origine commune. L'époque de Pisistrate voit la mise en œuvre de mesures visant à développer le culte dionysiaque (embellissement du temple du dieu, qui devient aussi le siège du théâtre, institution des jeux, annexion de la cité d'Éleuthères, transport du xoanon de Dionysos à Athènes, présence dionysiaque accrue sur les vases). Elles partagent avec les Panathénées un caractère politique et social marqué, mais s'en distinguent par une série de traits qui leur confère une place à part entière dans le système religieux athénien. Les jeux dramatiques qui se déroulaient à Athènes à cette occasion sont leur composante centrale et essentielle. Culte public au caractère particulièrement démonstratif, les Grandes Dionysies comprennent entre autres, une grande procession où la statue de Dionysos Eleuthereus est conduite d'une eschara située le long de la route d'Athènes à Eleuthères jusqu'à son théâtre athénien. Cette procession est aussi une grande parade dans laquelle la cité d'Athènes se donne à voir dans tout l'éventail de sa diversité qui se veut le reflet de sa cohésion sociale. Les membres de la société athénienne (auxquels s'ajoutent des groupes marginaux comme les éphèbes, - porteurs de la statue de Dionysos -, les orphelins de guerre, les étrangers, les métèques, les esclaves, les proxènes et les femmes) y jouent chacun leur rôle, qui dans la procession, qui dans le public. Ainsi, les ambassadeurs, les esclaves et certaines femmes, qui ne sont là qu'en spectateurs, y tiennent leur place de témoins de cette cohésion sociale, confortant ainsi le sentiment de l'identité athénienne. Le renouvellement des rites dionysiaques peut être considéré comme le reflet d'une volonté politique de resserrer les liens entre campagne et ville; quant à l'intégration à la procession officielle de groupes généralement considérés comme marginaux (mineurs, vierges et femmes de métèques, esclaves et étrangers), il peut s'agir d'un moyen d'affaiblir l'aristocratie et, en la donnant en spectacle, de renforcer la cohésion sociale de l'Attique. Chacun des groupes évoqués par les textes antiques fait ainsi l'objet d'une étude approfondie qui le situe à sa juste place sur la palette de groupes sociaux qui constituent l'Athènes du Ve $\mathrm{s}$. En ce qui concerne les orphelins de guerre, ils étaient, à Thasos, également mis à l'honneur en rece- 
vant une panoplie, non aux Dionysies, mais aux concours organisés lors des Héracleia ( $c f$. la Stèle des Braves de Thasos). Je me demande donc si ces groupes marginaux pointés par l'A. comme étant spécifiques aux fêtes dionysiaques le sont vraiment et si le sujet n'aurait pas requis d'élargir la perspective, en sortant du monde trop étroitement dionysiaque.

Une conception de la politique et une certaine vision du monde sont les deux faciès de l'identité athénienne qui s'expriment ainsi à travers ces quatre fêtes. Un même système de valeurs constitue le noyau de ces quatre fêtes qui peuvent ainsi se corréler l'une l'autre. La référence au vin est l'un des dénominateurs communs aux Anthestéries, aux Lénéennes et aux Dionysies aux champs, mais elle n'apparait pas clairement dans les Grandes Dionysies. D'autre part, toutes ces fêtes n'accueillant pas les mêmes marginaux, l'A. montre qu'un système de compensations devait réguler la participation aux fêtes de ces groupes. Ainsi, si les Lénéennes ne comportent pas d'étrangers, elles admettent la participation exceptionnelle de métèques dans la composition des chœurs; d'autre part, les étrangers participent aux Grandes Dionysies, mais les métèques n'y sont pas admis dans les chœurs. L'ouverture sur les étrangers, les esclaves, le monde extérieur est maximale dans les Grandes Dionysies, mineure dans les Lénéennes, mais est compensée par un rôle exceptionnel donné aux métèques et aux étrangers présents à Athènes. L'étude des fêtes et des systèmes rituels qui les sous-tendent (dont la base commune est la suspension de l'ordre) montre qu'à l'époque classique les rites dionysiaques sont parfaitement intégrés dans les structures politiques, religieuses et sociales de la cité grecque.

L'éclairage très particulier que donne l'A. à ces quatre fêtes dionysiaques n'épuise pas, à mon avis, le sujet complexe et plein de zones d'ombre par manque de témoignages, des fêtes dionysiaques. L'ouvrage ne prend pas en considération la documentation figurée et sans doute peut-on le regretter car les images, mêmes bien connues, auraient probablement pu apporter des informations complémentaires à son propos. La délicate question des Anthestéries/Lénéennes, de l'ouverture des jarres et des images montrant des rituels féminins devant les jarres aurait mérité une nouvelle approche iconographique. Il n’en reste pas moins qu'il apporte un éclairage nuancé sur la société athénienne de l'époque classique et qu'il constitue une somme d'informations qui marquera les études dionysiaques.

Isabelle TASSIGNON (FUNDP - Namur)

Laurent BriCAUlT, Isis, Dame des flots, Liège, CIPL, 2006. 1 vol. $18 \times 26 \mathrm{~cm}$, 244 p. 3 cartes, 80 fig. (Aegyptiaca Leodiensia, 7).

Cette enquête met en avant en sept chapitres les liens qui unissent Isis, et dans une moindre mesure Sarapis, à l'élément marin. Usant de nombreux documents, tant textuels qu'iconographiques, et souvent méconnus, sinon inédits, L. Bricault présente avec clarté les divers et délicats problèmes que soulève cette importante prérogative de la déesse. Le titre choisi témoigne déjà de la précision et de l'ingéniosité dont fait preuve l'A. tout au long de son analyse. Isis est bien une Dame des flots, mais pas, à proprement parler, une déesse marine, puisqu'elle ne vit pas dans la mer.

Il est d'abord question des origines de cet aspect d'Isis (p. 13-36). Dans l'Égypte pharaonique, seuls Amon et Hathor paraissent avoir été parfois considérés comme des maitres des flots. Isis n'a alors que des liens ténus avec l'élément aquatique, qu'il soit fluvial ou maritime. Plutôt que les Égyptiens, ce sont les Phéniciens de Byblos, voire les Grecs de Naucratis, qui pourraient avoir indirectement joué un rôle dans l'acceptation d'Isis en tant que déesse maritime. Ce trait n'apparait toutefois réellement qu'au début du $\mathrm{III}^{\mathrm{e}}$ siècle av. J.C. sous l'influence d'Arsinoé II et de son amiral, Callicratès de Samos. Une fresque de 